\section{Teleki to Leakey}

Andrew Hill

Koobi Fora: Researches into Geology, Palaeontology, and Human Origins.

Volume 2 The Fossil Ungulates: Proboscidea, Perissodactyla, and Suidae.

Edited by J.M. Harris.

Oxford University Press: 1983. Pp.320. $£ 45, \$ 95$.

IT WAS less than a hundred years ago that Europeans first looked upon Lake Turkana, when Count Samuel Teleki von Szek and his fellow traveller Ludwig von Hohnel came across it on 6 March 1888.

"An entirely new world was spread before our astonished eyes', wrote von Hohnel on seeing the huge expanse of water in the deserts of northern Kenya.

After Teleki few Westerners visited the lake. Sir Vivian Fuchs led an expedition there in the 1930s, and in 1943 Louis Leakey was told of the existence of fossils by a District Commissioner, but was denied access at that time. It was left to his son Richard to rediscover the palaeontological potential of the area in 1967. He began the Koobi Fora Research Project in 1968 and it still continues. While it may be hyperbolic to suggest an entirely new world has been spread before our astonished eyes as a result, certainly the research of the Project has changed our view of palaeoanthropology in significant ways.

In 1968 only three specimens of early hominid were known from Kenya. The Koobi Fora expedition has changed that, for a great deal of material has come from Lake Turkana, most of it discovered by an expert group of Kenyan fossil prospectors led by Kamoya Kimeu. Now the list of hominid fossils exceeds 150 for Koobi Fora alone, and the project has spawned as many publications. This monograph, edited and largely written by John Harris, is the most recent of them. It is dedicated to the memory of the late Sonia Cole, the technical editor of this and the first Koobi Fora volume, who in many ways made notable contributions to palaeoanthropology in Africa.

Aside from the sheer abundance of hominid specimens, what are the main achievements of the Project? Koobi Fora is one of three large sites in eastern Africa that document the radiation of hominids between two and one million years ago. Olduvai in Tanzania provided the first evidence, an assortment of specimens comprising two new taxa, Australo-

Pluto's Republic, Sir Peter Medawar's foray into "the intellectual underworld", has recently been published in paperback. The book first appeared in 1982, and in his review (Nature 300, 118; 1982) Stuart Sutherland wrote of Medawar's "Johnsonian thump of common sense"'. Publisher of the paperback is Oxford University Press; price is $£ 4.95$. pithecus boisei and the originally more controversial Homo habilis. It also produced the earliest complete assemblages of lithic culture. Work near the Omo River in southern Ethiopia resulted in important collections of vertebrate fossils, and a series of volcanic tuffs through the succession made it possible to establish a reliable temporal framework by potassium-argon dating. Although numerically abundant, the hominid finds were not spectacular, as sedimentary conditions in that area favoured mainly the preservation of isolated teeth.

Koobi Fora provided more complete specimens. Amongst them are good examples of Australopithecus boisei, enabling us to appreciate the high degree of sexual dimorphism and range of variation in this interesting creature. There are specimens that appear to confirm the reality of Homo habilis, and yet others that probably represent a third species. This might explain some of the earlier interpretative problems within the Olduvai sample. Another step was the recognition at Koobi Fora of the great antiquity in Africa of Homo erectus. This is the species generally thought to be immediately ancestral to our own, and is also the first hominid found outside Africa. Most specimens from the Far East and Europe, though, are more recent than one million years, whereas some from Koobi Fora are half-a-million years older. This makes it possible that even Homo erectus originated in Africa rather than elsewhere.

A theory being pushed with some vigour until a few years ago was that once hominids and material culture were established there was room ecologically for only one species. The finding of Homo erectus at this level, where it was contemporary with at least Australopithecus boisei, successfully demolished that notion. Hominid post-cranial bones were also found, resulting in a better understanding of locomotion in these early groups.

A variety of other research has been conducted under the auspices of the expedition. Some of this is general and basic, for example that on evolutionary theory using the excellent record of fossil freshwater gastropods. Other work, such as the investigations of the geology, palaeoecology and taphonomy of recent and fossil bone assemblages, is essential to understanding the environmental and time context of the hominids. Some deals fairly directly with hominid activities. Glynn Isaac, who with Richard Leakey is the Project's co-director and co-editor of this Koobi Fora monograph series, has led a team of archaeologists which has described two successive patterns of early stone tool technology that comprise distinctive facies within the Oldowan Industrial Complex. The team has also done much valuable work on principles of site interpretation.

Although the stone tools and the bits and pieces of old human skeletons form the glamorous treasures of palaeoanthropology, over 6,000 fossils of a wide range of other vertebrates have also been collected. These span the period from four to one million years ago, and are intrinsically important for what they tell us about the origins and evolution of certain taxonomic groups world wide, and also for their contribution to understanding the environments and ecology in which the hominids evolved. John Harris is the coordinator of palaeontology in the Project, and the present volume is the first of two dealing with the fossil ungulates. It is not designed for casual reading. Five of the seven chapters are detailed taxonomic treatments of the Deinotheriidae, Elephantidae (by Michel Beden), Rhinocerotidae, Equidae (by Vera Eisenmann), and Suidae, with complete listings and field locations of specimens. An introductory chapter gives the history of exploration and stratigraphical interpretation; a concluding one discusses correlation with other sites, biozonation and environmental change.

Correlation is one of the crucial questions to which the palaeontological information relates. The initial radiometric dating at Koobi Fora led to the impression that the artefacts there, and the fossil material belonging to the genus Homo, were the oldest in the world. Palaeoanthropologists traditionally have a proprietary attitude to their finds, and if they can possess the oldest they are in a very attractive position. It is attractive for publicity and therefore funding if nothing else, and so is a position they are understandably loath to give up. Such resistance was shown at Koobi Fora. Evidence was accumulating from the fauna which made clear an apparent time disjunction between there and other sites, particularly the Omo, only a few tens of kilometres distant. A number of ingenious explanations were devised to account for this disparity, before fresh dating, and more detailed work on the quite complicated stratigraphy, showed that earlier correlations were simply wrong. The hominids and artefacts are comparable in age to the oldest of their kind found elsewhere in East Africa, such as those from Olduvai. While slightly tarnishing the tinsel, this does little to reduce the area's scientific appeal.

Palaeontology is more than a description of biological events that just happen to have taken place in the past, and more than a simple narrative of phylogenetic histories. This present volume is an important work, and should in no sense be seen as a mere epitaph to these departed beasts. Rather it provides the basic data which, when taken with comparable information from other sites, could answer fundamental biological questions in evolution and ecology that neontology's perspective, limited as it is to extant creatures and communities, is too narrow to solve. $\square$

Andrew Hill is a Research Fellow at Harvard University. 